\section{CS-25 AN ANALYSIS OF HYDROXYCHLOROQUINE TREATMENT RESPONSE BY SEX IN PATIENTS WITH SYSTEMIC LUPUS ERYTHEMATOSUS}

D Koh, K Beattie, K Legault, G loannidis, M Matsos*. Department of Medicine, MMaster University, Hamilton, Ontario, Canada

\subsection{6/lupus-2018-Ism.60}

Background Systemic lupus erythematosus (SLE) has traditionally been viewed as a disease of women since the ratio of affected women to men is 9:1. Historically, men with SLE were thought to have more severe disease and worse prognosis than women, however, a 2013 review did not find compelling evidence that damage, disease activity or mortality is affected by sex. It concluded that the only consistent sexbased differences in SLE were lower rates of select disease manifestations such as alopecia.

Given this, it is plausible that the two sexes also respond to treatment differently, yet very few men have been included in clinical trials. Only $4 \%(n=9)$ of participants in published

Abstract CS-25 Table 1 Characteristics of the CaNIOS cohort treated with HCQ by sex

\begin{tabular}{|c|c|c|c|}
\hline & Total & Men & Women \\
\hline CaNIOS Patients & $n=499$ & $n=57$ & $n=442$ \\
\hline Age, mean $(S D)$ years & $33.6(17.9)$ & $26.2(16.4)$ & $34.6(17.9)$ \\
\hline Age at SLE diagnosis, mean (SD) years & $26.0(15.5)$ & $19.5(13.3)$ & $26.9(15.6)$ \\
\hline Disease duration, mean (SD) years & $7.2(7.8)$ & $6.2(6.5)$ & $7.3(7.9)$ \\
\hline Alcohol Intake, N (\%) & $381(76.4)$ & $38(66.7)$ & $343(77.6)$ \\
\hline None & $290(76.1)$ & $26(68.4)$ & $264(77.0)$ \\
\hline 1-6 drinks/week & $76(19.9)$ & $12(31.6)$ & $64(18.7)$ \\
\hline 7-14 drinks/week & $13(3.4)$ & 0 & $13(3.8)$ \\
\hline$\geq 15$ drinks/week & $2(0.5)$ & 0 & $2(0.6)$ \\
\hline Smoking, N (\%) & $415(83.2)$ & $46(80.7)$ & $369(83.5)$ \\
\hline Never & $322(77.6)$ & $38(82.6)$ & $284(77.0)$ \\
\hline$\leq 5$ cigarettes/day & $16(3.9)$ & $2(4.3)$ & $14(3.8)$ \\
\hline$>5$ cigarettes/day & 77 (18.6) & $6(13.0)$ & $71(19.2)$ \\
\hline Number of DMARDs, $\mathrm{N}(\%)$ & $499(100)$ & $57(100)$ & $442(100)$ \\
\hline None & $281(56.3)$ & $31(54.4)$ & $250(56.6)$ \\
\hline 1 DMARD & $197(39.5)$ & $24(42.1)$ & $173(39.1)$ \\
\hline 2 DMARDs & $19(3.8)$ & $2(3.5)$ & $17(3.8)$ \\
\hline 3 DMARDs & $2(0.4)$ & 0 & $2(0.5)$ \\
\hline Prednisone, N (\%) & $218(43.7)$ & $32(56.1)$ & $186(42.1)$ \\
\hline None & $1(0.5)$ & $0(0)$ & $1(0.2)$ \\
\hline Low (<7.5 mg PO daily) & $82(37.6)$ & $9(28.1)$ & $73(39.2)$ \\
\hline Medium (7.5-15 mg PO daily) & $77(35.3)$ & $14(43.8)$ & $63(33.9)$ \\
\hline High (>15 mg PO daily) & $58(26.6)$ & $9(28.1)$ & $49(26.3)$ \\
\hline Ethnicity & $498(99.8)$ & $57(100)$ & $441(99.8)$ \\
\hline Aboriginal & $34(6.8)$ & $5(8.8)$ & $29(6.6)$ \\
\hline Arab/Middle Eastern & $11(2.2)$ & $2(3.5)$ & $9(2.0)$ \\
\hline Asian & 99 (19.9) & $20(35.1)$ & $79(17.9)$ \\
\hline Black & $39(7.8)$ & $2(3.5)$ & $37(8.4)$ \\
\hline Jewish & $6(1.2)$ & 0 & $6(1.4)$ \\
\hline Latin/Hispanic & $13(2.6)$ & $1(1.8)$ & $12(2.7)$ \\
\hline Pacific Islander & 0 & 0 & 0 \\
\hline White & $291(58.4)$ & $27(47.4)$ & $264(59.9)$ \\
\hline Don't know/Don't wish to answer & $5(1.0)$ & 0 & $5(1.1)$ \\
\hline Annual Household Income, N (\%) & $371(74.3)$ & $43(75.4)$ & $328(74.2)$ \\
\hline$<\$ 15000$ & $34(9.2)$ & $7(16.3)$ & $27(8.2)$ \\
\hline$\$ 15,000-\$ 29999$ & $45(12.1)$ & $8(18.6)$ & $37(11.3)$ \\
\hline$\$ 30,000-\$ 49999$ & $88(23.7)$ & $6(14.0)$ & $82(25.0)$ \\
\hline$>\$ 50000$ & $204(55.0)$ & $22(51.2)$ & $182(55.5)$ \\
\hline Highest Education, N (\%) & $308(61.7)$ & $27(47.4)$ & $281(63.6)$ \\
\hline Did not complete high school & $61(19.8)$ & $9(33.3)$ & $52(18.5)$ \\
\hline High school diploma & $24(7.8)$ & $3(11.1)$ & $21(7.5)$ \\
\hline Vocational/technical school & $54(17.5)$ & $3(11.1)$ & $51(18.1)$ \\
\hline Some college, no degree & $49(15.9)$ & $4(14.8)$ & $45(16.0)$ \\
\hline College degree & $104(33.8)$ & $7(25.9)$ & $97(34.5)$ \\
\hline Graduate/professional degree & $16(5.2)$ & $1(3.7)$ & $15(5.3)$ \\
\hline DI score, mean(SD) & $0.8(1.3)$ & $1.1(1.8)$ & $0.8(1.2)$ \\
\hline SLEDAI 2K score, mean(SD) & $4.5(4.7)$ & $3.7(4.9)$ & $4.6(4.7)$ \\
\hline
\end{tabular}

DMARD=disease modifying antirheumatic drug. 
randomized controlled trials examining the impact of hydroxychloroquine (HCQ) on SLE were men. Thus, our objective was to examine the disease activity of both women and men with SLE treated with HCQ. In a cross-sectional analysis, we hypothesized that men with SLE would have higher disease activity than women based on average SLE Disease Activity Index (SLEDAI) 2K and Damage Index (DI) scores.

Methods Patient information was collected from the Canadian Network for Improved Outcomes in SLE (CaNIOS) database. All patients had an SLE diagnosis confirmed using the American College of Rheumatology (ACR) criteria. Inclusion in this study required that patients be $\geq 18$ years of age and be currently taking any prescribed dosage regimen of HCQ. Data for these analyses were collected at entry into CaNIOS. Descriptive characteristics included health habits, ethnicity, immunosuppressant medication usage and SLEDAI 2K 10 day and DI scores. Sex differences in SLEDAI $2 \mathrm{~K}$ and DI scores were analyzed using multivariate regression analyses controlling for prednisone use, current age, age at SLE onset and disease duration.

Results A total of 499 patients ( $n=57$ men, $n=442$ women) treated with HCQ were included (table 1). Men appeared to have slightly lower SLEDAI $2 \mathrm{~K}$ scores and higher DI scores than women, and had slightly higher use of prednisone. However, regression analyses revealed no differences in SLEDAI $2 \mathrm{~K}$ scores between sexes, yet current age was significant $(\beta=0.228, p=0.021)$. DI scores were significantly associated with current age $(\beta=0.349, p=0.001)$ and disease duration $(\beta=0.170, p=0.002)$ but not sex.

Conclusions In contrast to our hypothesis, we found no sex differences in disease activity or damage index between men and women. Results of this study are limited by lack of control for HCQ dose and duration of use which will be addressed in future analyses. Future analyses will investigate potential sex differences in longitudinal changes in SLEDAI and DI scores in response to $\geq 3$ months of HCQ therapy.

\section{CS-26 WHAT DO WE MEAN WHEN WE SAY SLE?}

${ }^{1}$ Linjia Jia, ${ }^{2}$ Ecem Sevim, ${ }^{2}$ Medha Barbhaiya, ${ }^{2}$ Michael D Lockshin*. 'Weill-Cornell Medicine, New York, New York, USA; ${ }^{2}$ Barbara Volcker Center, Hospital for Special Surgery, New York, New York, USA

\subsection{6/lupus-2018-Ism.61}

Background Although lupus spectrum illness contains at least four variants (antibody only, undifferentiated autoimmune disease, typical SLE, and overlap SLE), clinical, epidemiological, and basic science publications do not use distinguish among these groups when they speak of SLE, thereby muddling interpretation of the results.

Methods We systematically reviewed clinical, epidemiological, and basic science publications from the last 5 years to determine definitions they used for SLE.

Results Of 366 papers on human SLE, 197 were suitable for analysis. Of these, 32 were basic/translational, 151 clinical trials and studies, and 14 epidemiological. Between $66 \%$ and $79 \%$ of papers specified or implied use of ACR criteria, of which $0 \%-16 \%$ also required presence of anti-DNA or antiSm antibody. 19\%-29\% of papers specified exclusion criteria, of which 10\%-14\% specifically excluded patients with overlap disease. Disease activity was considered in $14 \%-22 \%$ and stable disease was required in 7\%-14\% of papers. $0 \%-3 \%$ of papers considered disease duration (table 1).
Of 132 studies on mouse SLE, 24\% use NZB variants, $36 \%$ MRL-lpr variants and 39\% used variants/induced illness in $\mathrm{C} 57 \mathrm{Bl} / 6 \mathrm{~J}$ mice. Definition of SLE required presence of anti-DNA antibody in $47 \%$ of studies, ANA in 10\%, proteinuria in $39 \%$ and immunohistochemistry (usually renal) in $57 \%$. $15 \%$ of papers defined disease onset $\leq 8$ weeks, $15 \% 8-16$ weeks, and $57 \%$ did not indicate time of disease onset. The fact that the paper spoke of animal SLE was indicated in the titles of $46 \%$ of papers.

\begin{tabular}{lllll}
\multicolumn{1}{l}{ Abstract CS-26 Table } & \multicolumn{7}{l}{} \\
\hline Type of study (N) & Basic (32) & Clinical (151) & Epidem (14) & All (197) \\
\hline ACR Criteria only & 69 & 66 & 79 & 67 \\
aDNA or aSm specified & 13 & 9 & 0 & 9 \\
Exclusion criteria specified & 19 & 31 & 29 & 29 \\
Exclusion overlap specified & 13 & 10 & 14 & 11 \\
Activity SLEDAI or BILAG & 22 & 16 & 14 & 17 \\
Stable disease specified & 13 & 7 & 14 & 9 \\
Duration specified & 0 & 3 & 0 & 3 \\
\hline
\end{tabular}

Criteria used in 197 studies on human SLE, indicating criteria for diagnosis, inclusions, exclusions, serology, activity, and disease duration.

Conclusion Publications on SLE use a wide variety of definitions, inclusion, and exclusion criteria and often use the term SLE without specifying animal or human. Consensus definitions and stratification rules are necessary to obtain greater homogeneity in studies of SLE. (We will propose methods by which greater consensus can be obtained.)

Acknowledgements Supported by the Barbara Volcker Center and the Weill Cornell Medicine AOC program.

\section{CS-27 MORTALITY AMONG PATIENTS WITH SYSTEMIC LUPUS ERYTHEMATOSUS HOSPITALIZED WITH SEPSIS VARIES WIDELY AMONG U.S. HOSPITALS}

${ }^{1}$ Michael M Ward*, ${ }^{2}$ Maria Tektonidou, ${ }^{1}$ Abhijit Dasgupta. ${ }^{1}$ National Institute of Arthritis and Musculoskeletal and Skin Diseases, NIH, U.S; ${ }^{2}$ First Department of Propaedeutic Internal Medicine, Joint Academic Rheumatology Program, Medical School, National and Kapodistrian University of Athens, Athens, Greece

\subsection{6/lupus-2018-Ism.62}

Background Sepsis is a serious infection that is often difficult to treat. We investigated if the risk of mortality in patients with systemic lupus erythematosus (SLE) hospitalized with sepsis varies among hospitals.

Methods We used the National Inpatient Sample (2002-2011) to obtain data on outcomes of adults age 18 to 64 with SLE who were admitted with a diagnosis of sepsis. We included 424 hospitals that had at least 5 hospitalizations of patients with SLE and sepsis. We abstracted data on demographic features, diagnoses, and mortality for these patients and for patients without SLE hospitalized with sepsis at the same set of hospitals. We used machine learning methods to derive the expected risk of in-hospital mortality for each patient, based on demographic features, insurance status, comorbidities, and diagnoses related to organ failure. We then computed the observed/expected ratio for in-hospital mortality separately for SLE and non-SLE patients by hospital. To further adjust for case-mix, the ratio of these two ratios (RR) was used to 